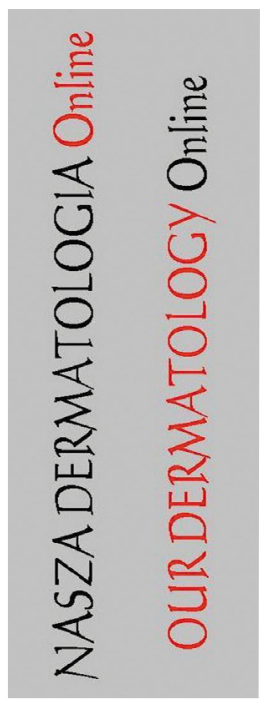

\title{
SUBUNGUAL GLOMUS TUMOUR SUBUNGUAL GLOMUS TUMOUR
}

\author{
Rohini Mathias ${ }^{1}$, Sharad Ramdas ${ }^{2}$, Renu G. Varghese ${ }^{3}$ \\ ${ }^{1}$ Department of Dermatology, Venereology and Leprology, Pondicherry \\ Institute of Medical Sciences, Pondicherry, India \\ ${ }^{2}$ Department of Plastic Surgery, Pondicherry Institute of Medical Sciences, \\ Pondicherry, India \\ ${ }^{3}$ Department of Pathology, Pondicherry Institute of Medical Sciences, \\ Pondicherry, India
}

Corresponding author: Dr. Iffat Hassan

hassaniffat@gmail.com

A $35 y$ year old housewife presented with a two year history of paroxysmal excruciating pain over the distal end of left ring finger with aggravation of pain on minimal pressure and exposure to cold. There was no history of preceding trauma. Analgesics, antibiotics and anti-ischemic drugs had not provided any relief. Cutaneous examination revealed a mild swelling of the proximal nail fold with a subtle blue discoloration over the proximal nail bed (Fig. 1, 2).

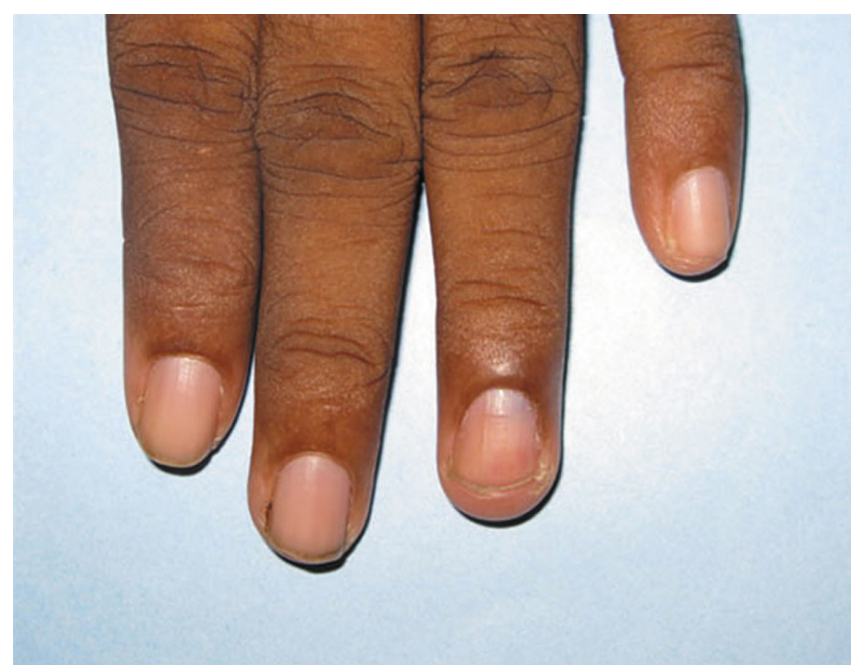

Figure 1. Mild swelling of the proximal nail fold with a subtle blue discoloration over the proximal nail bed

Hildreth sign and Love test were positive. Routine investigations and an X-Ray of the finger revealed no abnormalities. Histopathological examination of the excised lesion revealed a well circumscribed neoplasm consisting of sheets of round cells with punched out nuclei and pale eosinophilic cytoplasm (glomus cells) surrounding endothelium lined vascular spaces, features which confirmed the diagnosis of a subungual glomus tumor (Fig. 3, 4).

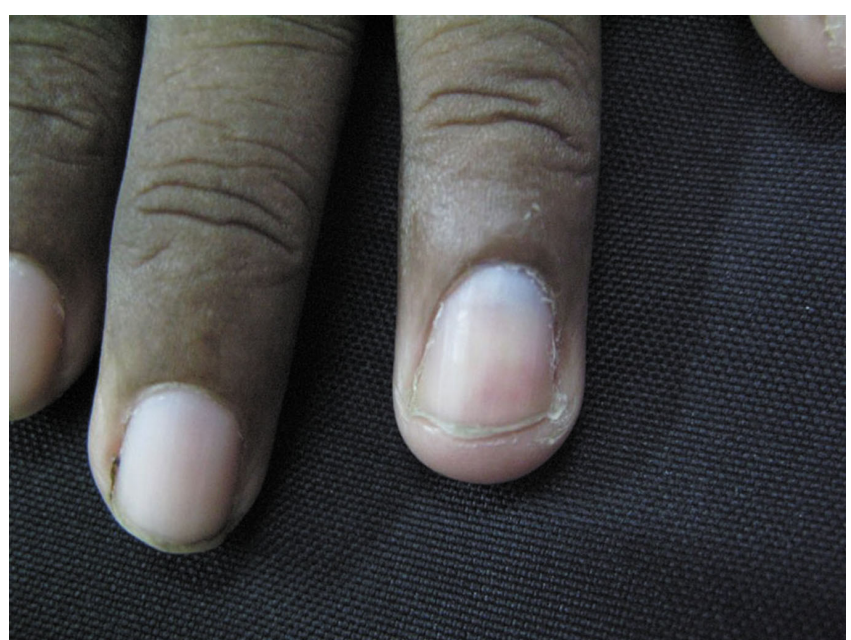

Figure 2. Mild swelling of the proximal nail fold with a subtle blue discoloration over the proximal nail bed

Glomus (Latin- ball of thread) tumors, are uncommon, painful hamartomas composed of perivascular cells resembling modified smooth muscle cells of the normal glomus body. Glomus bodies are intradermal arteriovenous shunts with a thermoregulatory function concentrated in the finger and toe tips especially in the subungual region. Glomus tumors may be single or multiple, the former being more common. The most common site of occurrence is the hand which accounts for $75 \%$ of all cases, subungual lesions predominating. A classic triad of paroxysmal pain, cold sensitivity and point tenderness has been described. Love's test consists of eliciting point tenderness with a fine instrument such as the tip of a pencil or pinhead.

Hildreth's sign is the disappearance of pain after a tourniquet is applied on the extremity, proximal to the lesion. Dermoscopy has been used pre- and intra-operatively to delineate the tumor. 
Depending on the predominant cell type, which may be glomus cells, vascular structures or smooth muscle cells, glomus tumors are classified as solid glomus tumor, glomangioma and glomangiomyoma respectively.

Imaging techniques include ultrasonography and high resolution MRI. Doppler studies have been used to assess tumor vascularity. X-Rays are less helpful. Complete surgical excision (subungual, lateral or volar approach) is the treatment of choice. Multiple tumors may be managed with sclerotherapy or laser ablation (CO2, argon and pulsed dye lasers).

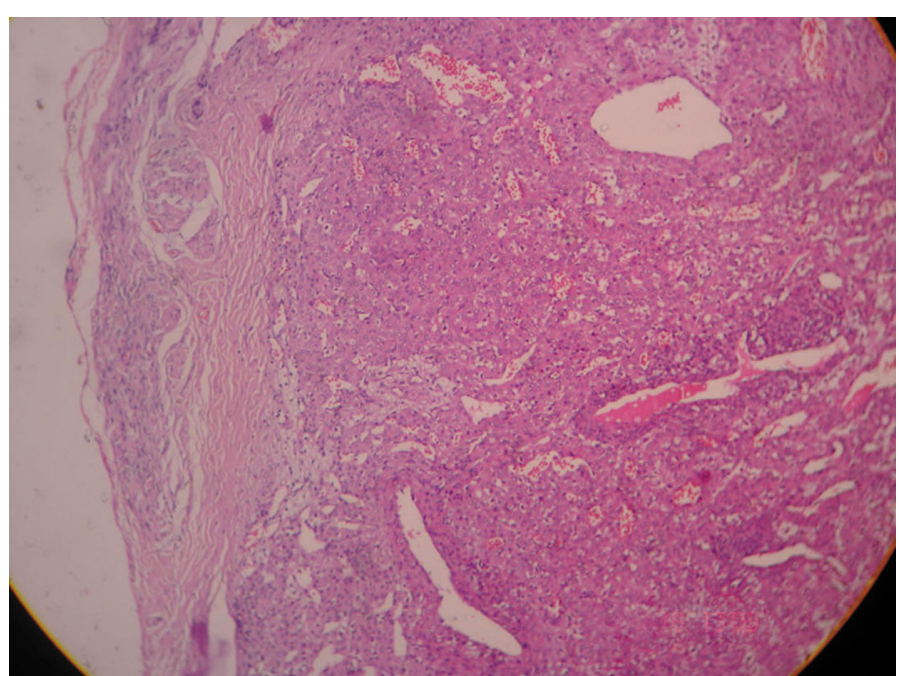

Figure 3. Well circumscribed neoplasm consisting ofsheets of round cells with punched out nuclei and of round cells with puns paleeosinophilic cytoplasm (glomus cells) surrounding endothelium lined vascular spaces

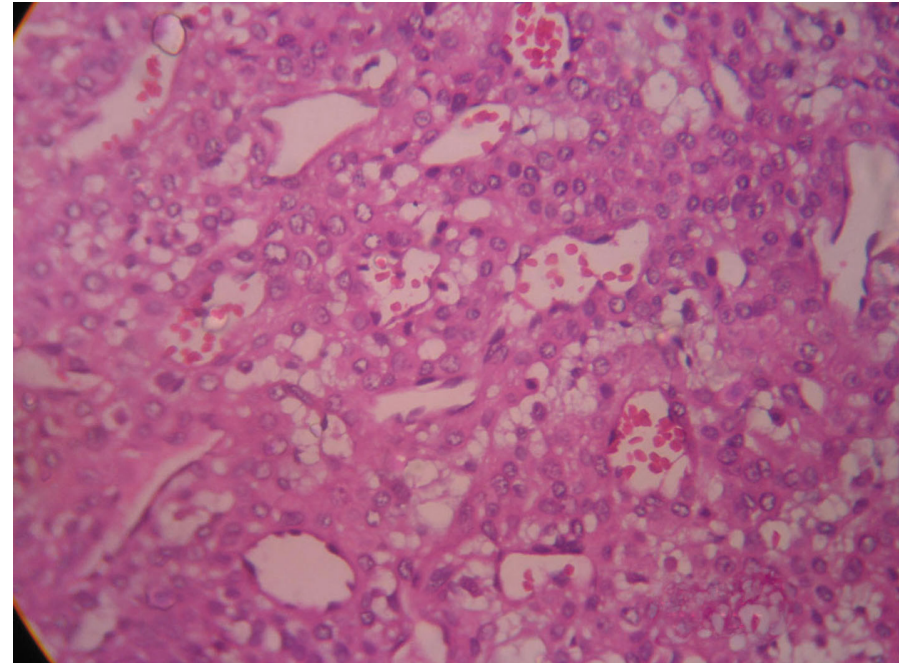

cytoplasm (glomus cells) surroundingendothelium lined vascular spaces

\footnotetext{
Copyright by Rohini Mathias, et al. This is an open access article distributed under the terms of the Creative Commons Attribution License, which
} permits unrestricted use, distribution, and reproduction in any medium, provided the original author and source are credited. 